\title{
Enhanced Acetylene Emission near the North Pole of Jupiter
}

\author{
PIERRE DROSSART ${ }^{*}, 1$ BRUNO BÉZARD, ${ }^{*}$ SUSHIL ATREYA $\dagger$ JOHN LACY,$\neq 1$ \\ EUGENE SERABYN, $\$, 1$ ALAN TOKUNAGA,",2 AND THÉRĖSE ENCRENAZ*
}

*Observatoire de Paris, section de Meudon, 92190 Meudon, France; †University of Michigan, Ann Arbor, Michigan; $\neq$ Department for Astronomy, University of Texas, Austin, Texas 78712; \$Department of Physics, University of California, Berkeley, California 94720; and "Institute for Astronomy, Honolulu, Hawaii 96822

Received June 3, 1985; revised February 24, 1986

\begin{abstract}
We report observations of acetylene emission lines near $13.3 \mu \mathrm{m}$ on Jupiter recorded at the NASA Infrared Telescope Facility in July 1984. A strong enhancement in the intensity of $R_{10}$ line of the $\nu_{5}$ band was recorded within a well-localized region coincident with the southern extension of footprint of the Io magnetic lines and with previous observations of localized enhanced emission of $\mathrm{CH}_{4}$ lines. The line intensity was fairly constant outside this "bright spot." Moreover, weak lines of the hot bands $2 \nu_{5}-\nu_{5}$, and $\left(\nu_{4}+\nu_{5}\right)-\nu_{5}$ were observed within the bright spot. From the field of view and the precision of the pointing, the zone of activity of the bright spot is found to be: latitude $=59 \pm 10^{\circ}$ and longitude $=178 \pm 10^{\circ}$ (System III, 1965). The location of the spot was found to be constant over a 3-day period. Two interpretations are proposed to explain these observations: (1) a variation of the $\mathrm{C}_{2} \mathrm{H}_{2}$ abundance and (2) an alteration of the thermal profile in the bright spot. Either may result from precipitation of charged particles near and below the Jovian homopause. 1986 Academic Press, Inc.
\end{abstract}

\section{INTRODUCTION}

Acetylene in the upper atmosphere of Jupiter was first observed by Ridgway (1974) and Combes et al. (1974). $\mathrm{C}_{2} \mathrm{H}_{2}$ is produced from $\mathrm{CH}_{4}$ via photochemical reactions. Once produced, $\mathrm{C}_{2} \mathrm{H}_{2}$ remains as a relatively stable molecule in the Jovian stratosphere (Gladstone, 1982; Strobel, 1974; Atreya and Romani, 1985) because $\mathrm{C}_{2} \mathrm{H}_{2}$ is reconstituted after its photodissociation into radicals (Atreya, 1984). In addition to the ground-based measurements of the abundance of $\mathrm{C}_{2} \mathrm{H}_{2}$ on Jupiter made by Ridgway et al. (1974), Combes et al. (1974), and Tokunaga et al. (1976, 1979), Voyager IRIS spectra at high spatial resolution dem-

\footnotetext{
1 Visiting Astronomer at the Infrared Telescope Facility which is operated by the University of Hawaii under contract from the National Aeronautical and Space Administration.

${ }^{2}$ Staff Astronomer at the Infrared Telescope Facility which is operated by the University of Hawaii under contract from the National Aeronautical and Space Administration.
}

onstrated the variability of the $\mathrm{C}_{2} \mathrm{H}_{2}$ abundance with latitude (Hanel et al., 1979; Maguire et al., 1984). Another product of the $\mathrm{CH}_{4}$ photochemistry, $\mathrm{C}_{2} \mathrm{H}_{6}$, is formed in the same altitude region as $\mathrm{C}_{2} \mathrm{H}_{2}$ in the Jovian atmosphere. Variations in the abundance of $\mathrm{C}_{2} \mathrm{H}_{2}$ with latitude have also been reported (Kostiuk et al., 1983), and enhancement in the south polar regions have been observed.

Modification of the stratospheric profile in the polar regions, due to the auroral electron precipitation, was suggested by the observation of high infrared brightness temperatures in the wing of the $\nu_{4}$ band of $\mathrm{CH}_{4}$ at $8 \mu \mathrm{m}$. (Caldwell et al., 1980, 1983). This infrared anomaly was found at a location similar to the Kostiuk et al. (1977) observations of $\mathrm{NH}_{3}$ line enhancement, the maximum of the UV auroral intensity (Broadfoot et al., 1979) and the location of the magnetic anomaly of Jupiter (Dessler, 1979). We present here observations of acetylene fundamental and hot band vibrational emission lines from the same region. 
TABLE I

Chronology of IRTF OBSERVATIONS

\begin{tabular}{ccccccc}
\hline Date & $\begin{array}{c}\text { Position on } \\
\text { the planet, } \\
\text { latitude/longitude }\end{array}$ & UT & $\begin{array}{c}\text { Wavenumber } \\
\left(\mathrm{cm}^{-1}\right)\end{array}$ & $\begin{array}{c}\text { Scan } \\
\text { number }\end{array}$ & $\begin{array}{c}\text { Integration } \\
\text { time } \\
(\mathbf{m i n})\end{array}$ & $\begin{array}{c}\text { Air } \\
\text { mass }\end{array}$ \\
\hline July 27 & Center & $8 \mathrm{~h} 20$ & 755 & 1 & 20 & 1.0 \\
$*$ & $57^{\circ} / 156^{\circ}$ & $8 \mathrm{~h} 59$ & - & 2 & 20 & 1.8 \\
$*$ & $57^{\circ} / 175^{\circ}$ & $9 \mathrm{~h} 30$ & - & 3 & 10 & 1.8 \\
$*$ & $57^{\circ} / 184^{\circ}$ & $9 \mathrm{~h} 45$ & - & 4 & 10 & 1.8 \\
$*$ & $69^{\circ} / 160^{\circ}$ & $9 \mathrm{~h} 55$ & - & 5 & 20 & 1.9 \\
July 30 & Center & $7 \mathrm{~h} 00$ & 745.8 & 6 & 20 & 1.0 \\
$*$ & $59^{\circ} / 193^{\circ}$ & $7 \mathrm{~h} 30$ & 745.8 & 7 & 10 & 1.9 \\
$*$ & $59^{\circ} / 186^{\circ}$ & $7 \mathrm{~h} 50$ & 745.8 & 8 & 10 & 2.1 \\
\hline
\end{tabular}

* Bright spot observation.

The observations are presented in Section II. Section III gives the synthetic calculations deriving the $\mathrm{C}_{2} \mathrm{H}_{2}$ abundance in the equatorial regions and at high north latitudes. Section IV gives a preliminary interpretation of the observations.

\section{OBSERVATIONS AND INSTRUMENTATION}

The observations were done with a cooled Fabry-Perot Grating Spectrometer (FPGS), which is described by Beck et al. (1982) and Serabyn (1984). The observations were made at the NASA Infrared Telescope Facility in Hawaii, in July 1984. The FPGS was previously used for the first detection of HCN on Jupiter (Tokunaga et al., 1981) and of ${ }^{13} \mathrm{C}^{12} \mathrm{CH}_{2}$ (Drossart et al., 1985b). The spectral resolution for the present observations was $0.03 \mathrm{~cm}^{-1}$ (full width at half maximum) and the noise equivalent power was about $4 \times 10^{-15} \mathrm{~W}$ $\mathrm{Hz}^{-1 / 2}$. The observations were made using a 3 -arcsec circular beam. The details of the observations are presented in Table I. As in Drossart et al. (1985a), the flux calibration is obtained from the continuum level of the Voyager IRIS data in the equatorial region. Temporal variations due to seasonal effects on Jupiter are found to be less than $2 \mathrm{~K}$ in the temperature of the upper troposphere (Orton, 1984) and IRIS spectra can be used for calibration. In the high north latitudes, the same absolute calibration can be used since the spectra were recorded within the same hour.

The wavenumber calibration was obtained from an acetylene cell at ambient temperature. The accuracy of the spectral positions is $\pm 0.005 \mathrm{~cm}^{-1}$ and the Doppler shifts due to the relative Earth-Jupiter motion and to the rotation of Jupiter were taken into account. The pointing accuracy introduces another uncertainty, because of the Doppler shift of the rotation, especially near the limb, of the order of $0.005 \mathrm{~cm}^{-1}$. Figures 1-3 show the recorded spectra. The parameters of the observed lines are given in Table II.

To search for variations in $\mathrm{C}_{2} \mathrm{H}_{2}$ emission lines, we looked for a region mentioned by Caldwell et al. $(1980,1983)$ as a region of enhanced infrared emission in the northern latitudes of Jupiter. The $\mathrm{C}_{2} \mathrm{H}_{2}$ line at $755.006 \mathrm{~cm}^{-1}$ was found to be enhanced in a region localized both in latitude and longitude. This region, which is referred to as the "bright spot" in the ensuing discussion, was found by scanning the Jovian disk. However, since the signal to noise ratio is too low for short spectra $(<10 \mathrm{~min})$ the entire disk of Jupiter could not be mapped at the spatial resolution of the FPGS.

This bright spot is characterized by (1) an enhancement of the fundamental lines of 


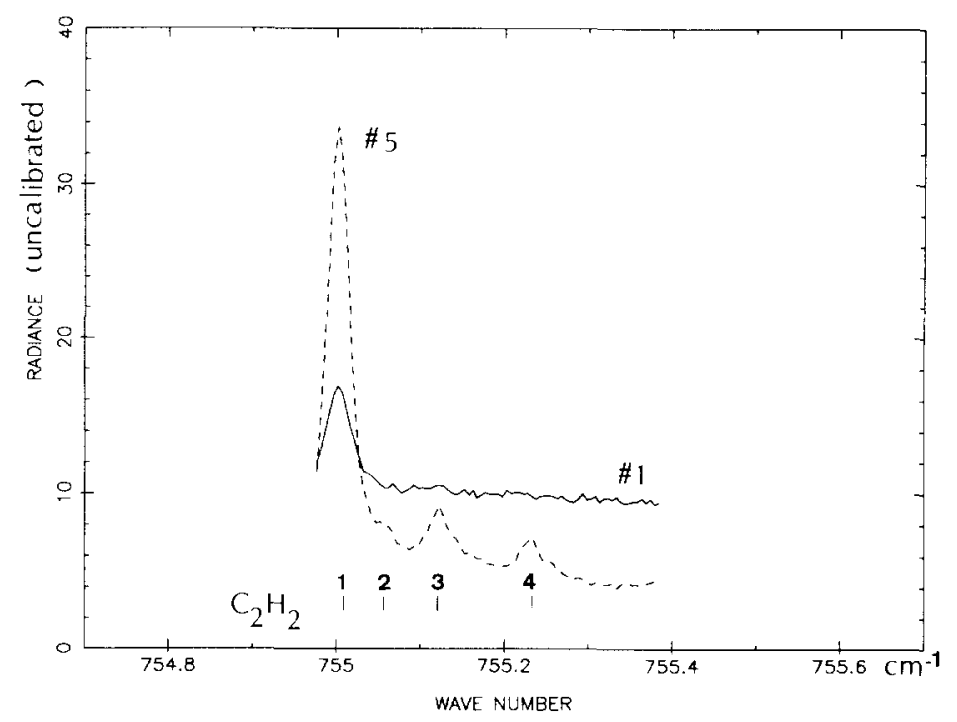

FIG. 1. Comparison of a spectrum at the center of the Jovian disk (1) and within the bright spot (5). The radiance scales (uncalibrated) are the same for both spectra. The labels of the curves conform to Table I. Line numbers refer to Table II.

the $\nu_{5}$ band of $\mathrm{C}_{2} \mathrm{H}_{2} ;(2)$ the appearance of hot band lines of $\mathrm{C}_{2} \mathrm{H}_{2}$. These bands are seen for the first time in the atmosphere of Jupiter.

The position of the bright spot on Jupiter was found to be constant between two observations done in a 3-day interval. The uncertainty in the position of the bright spot is found to be (approximately) $\pm 2^{\prime \prime}$. It was estimated by taking into account the field of view ( 3 " circular), the accuracy of the pointing during the recording of a spectrum $\left(\approx 1^{\prime \prime}\right.$ for $15 \mathrm{~min}$ integration), and by scanning across the spot. The position of the spot was obtained by scanning on the Jovian disk from limb to limb. The position of the limb can be determined with an accuracy of 1 ". Since the location of the bright spot was fairly well defined, we conclude that its dimension is smaller than the field of view, and it is located at the following position: latitude $=59 \pm 10^{\circ} ;$ longitude $=178 \pm 10^{\circ}$ (System III, 1965).

\section{SYNTHETIC CALCULATIONS}

The synthetic spectra are calculated using a line-by-line calculation. The model

TABLE II

\begin{tabular}{llcccc}
\hline Band & Identification & $\begin{array}{c}\text { Wavenumber } \\
\left(\mathrm{cm}^{-1}\right)\end{array}$ & $\begin{array}{c}\text { Energy of } \\
\text { lower level } \\
\left(\mathrm{cm}^{-1}\right)\end{array}$ & $\begin{array}{c}\text { Intensity } \\
(\text { at 300 K) } \\
\left(\mathrm{cm}^{-2} \mathrm{am}^{-1}\right)\end{array}$ \\
\hline 1 & $\nu_{5}$ & $R_{10}$ & 755.006 & 129.41 & 5.91 \\
2 & $2 \nu_{5}^{2 f}-\nu_{5}^{1 f}$ & $R_{10}$ & 755.068 & 795.29 & 0.175 \\
3 & $\left(\nu_{4}+\nu_{5}\right)^{2 \mathrm{e}}-\nu_{4}^{\text {le }}$ & $R_{9}$ & 755.126 & 718.71 & 0.585 \\
4 & $\left(\nu_{4}+\nu_{5}\right)^{0+}-\nu_{4}^{1 \mathrm{le}}$ & $R_{15}$ & 755.234 & 895.2 & 0.295 \\
5 & $2 \nu_{5}^{2 \mathrm{e}}-\nu_{5}^{\text {le }}$ & $R_{6}$ & 745.858 & 778.56 & 0.175 \\
\hline
\end{tabular}

Note. References: Hietanen and Kaupinnen (1981); Varanasi et al. (1983).

a The index is the value of $J$. 


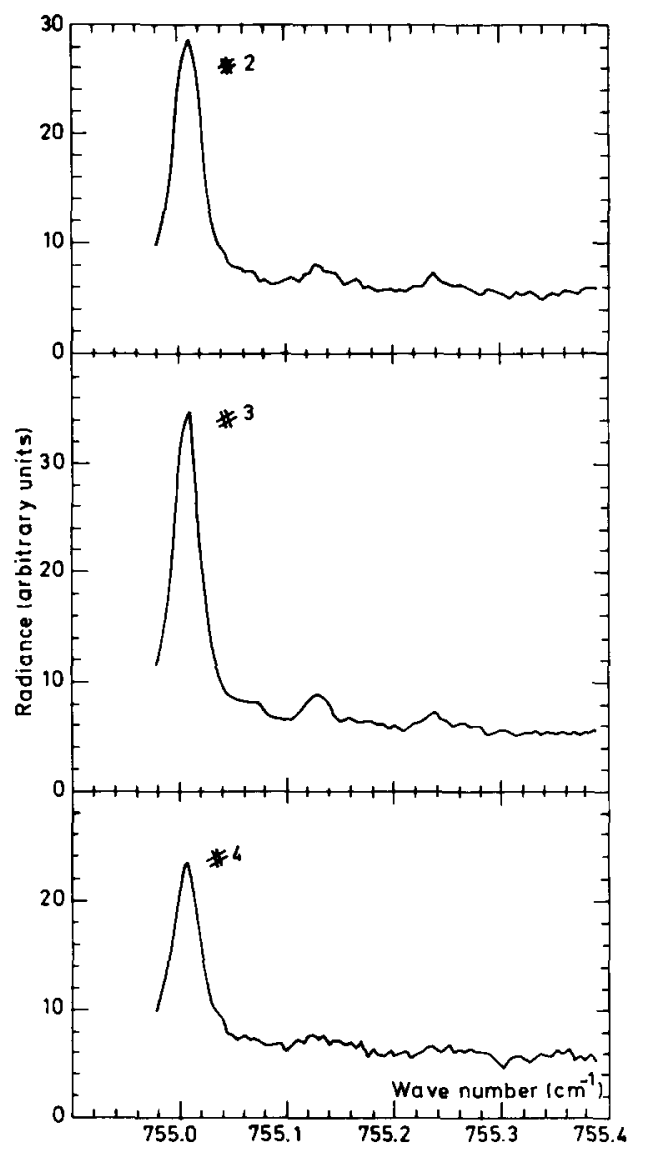

FIG. 2. Comparison of spectra recorded in the bright spot position at different time (the same scale is used in radiances). The labels of the curves correspond to Table I.

used is described in Drossart et al. (1985a, b) in the $850-$ to $900-\mathrm{cm}^{-1}$ range.

Retrieval of the thermal structure in the observed areas is the first step in an abundance determination. For that purpose, selections of Voyager IRIS spectra recorded at similar locations were used, neglecting variations of temperature since the Voyager encounter. Selection criteria upon the location of the IRIS field of view (latitude and longitude), the air mass, and the distance between the spacecraft and the center of the planet were applied. The choice of these constraints results from a tradeoff between the desired degree of homogeneity in the selection and the signal-to-noise ratio required for the thermal inversion algorithm. 128 spectra in the equatorial region were selected and used to calculate temperature profile 1 in Fig. 4. IRIS spectra with a field of view smaller than the field of view of the FPGS ( 3 " from Earth) were chosen. Although a homogeneous selection can be made at latitudes similar to that of the bright spot, no IRIS spectra fulfilling this selection criterion falls within the bright spot itself. A selection of nearby spectra were used to generate a second temperature profile (Fig. 4, profile 2). Figure 5 shows the emission in the $\nu_{5}$ band of $\mathrm{C}_{2} \mathrm{H}_{2}$ observed in these two IRIS selections.

Temperature profiles were retrieved from 1 to 600 mbar by the inversion method described in Gautier et al. (1977) using measurements at selected wavenumbers in the $\mathrm{S}(0)$ and $\mathrm{S}(1) \mathrm{H}_{2}$ lines and in the core of the $\nu_{4}$ band of methane. A methane to hydrogen mixing ratio equal to $2.3 \times 10^{-3}$ was assumed (Gautier et al., 1982; Gautier and Owen, 1983). The effective vertical resolution of the retrievals varies with altitude from 0.5 pressure scale height in the troposphere to 2 scale heights at the 1 mbar level; the formal errors due to instrumental noise are less than $1 \mathrm{~K}$ at any level.

The vertical profile of the $\mathrm{C}_{2} \mathrm{H}_{2}$ mixing ratio was assumed to be constant above the 20 mbar level and zero below, as in Drossart et al. (1985b). As the theoretical profiles (Strobel, 1983) give no better approximations at our spectral resolution $(0.03$ $\mathrm{cm}^{-1}$ ) and their lower cutoff limit is also somewhat arbitrary, they are not used.

Using these $\mathrm{C}_{2} \mathrm{H}_{2}$ and temperature profiles, the abundance of $\mathrm{C}_{2} \mathrm{H}_{2}$ is estimated to be (1) $8.0 \times 10^{-4} \mathrm{~cm} \cdot \mathrm{am}$ in the equatorial region $\left(\mathrm{C}_{2} \mathrm{H}_{2} / \mathrm{H}_{2}=1 \times 10^{-8}\right.$ above $\left.20 \mathrm{mbar}\right)$ and (2) $8.0 \times 10^{-3} \mathrm{~cm} \cdot$ am on the bright spot $\left(\mathrm{C}_{2} \mathrm{H}_{2} / \mathrm{H}_{2}=1 \times 10^{-7}\right.$ above $\left.20 \mathrm{mbar}\right)$. The comparison between synthetic and observed spectra is shown on Fig. 6 for the equatorial region, and in Fig. 7 for the bright spot. These values are estimated to be precise within a factor of 2 only, the 


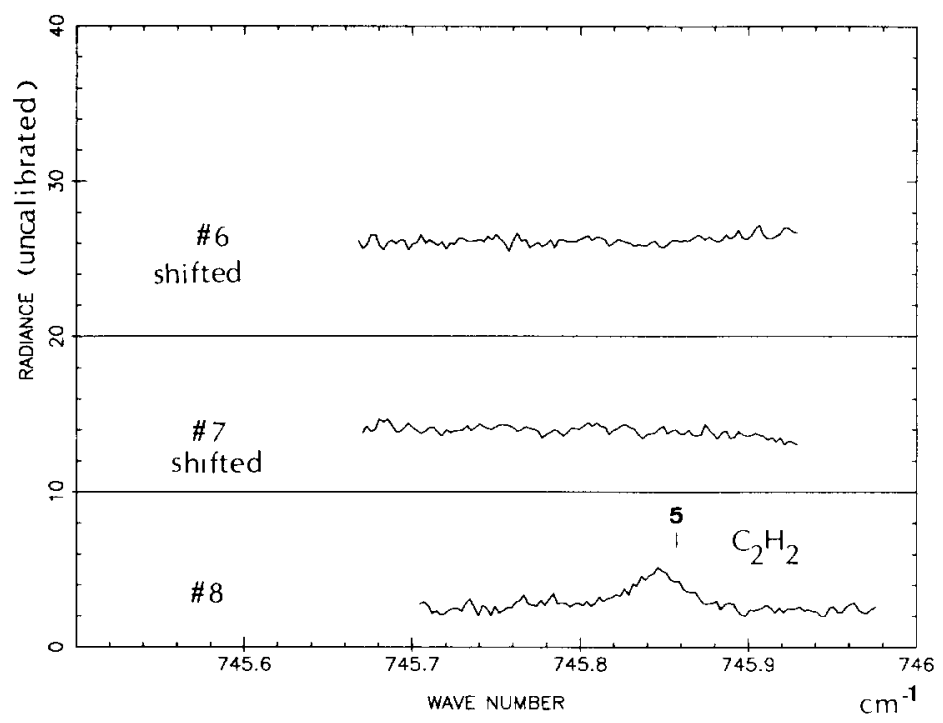

FIG. 3. Comparison of spectra at the same latitude but at different longitudes. Only spectrum 8 (labeled as in Table I) shows an acetylene hot band line at $745.86 \mathrm{~cm}^{-1}$; spectrum 7 is out side the bright spot. Spectrum 6 is recorded at the equator and is shown for comparison. Spectrum 6 and 7 are shifted on $y$-axis.

main uncertainties being the systematic errors inherent to the model (continuum, temperature profile and variation of abundance with height). The equatorial ratio is a factor of 3 lower than the Voyager determination of Maguire et al. (1984), and could be due to temporal variations, but direct comparison of the $\mathrm{C}_{2} \mathrm{H}_{2} / \mathrm{H}_{2}$ ratio are difficult because of

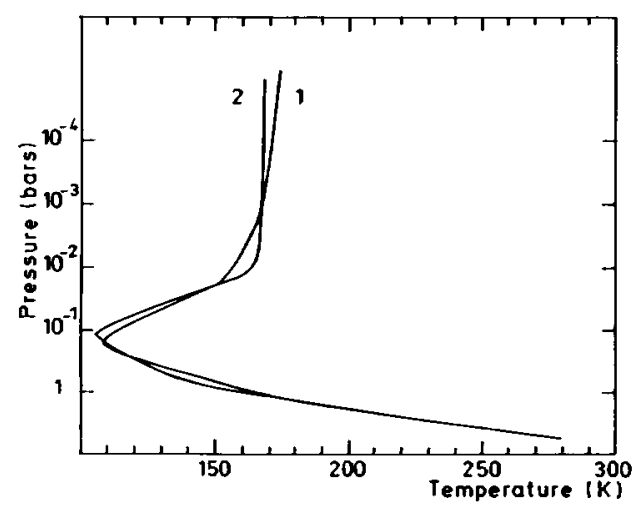

FIG. 4. Temperature profiles inverted from Voyager selection. (1) Equatorial selection: 128 IRIS spectra in the equatorial region (field of view smaller than FPGS); (2) selection at $60^{\circ} \mathrm{N}$ (but outside the bright spot): 53 IRIS spectra (field of view smaller than FPGS). the great uncertainties in the vertical distribution of acetylene. It is important to note that the temperature profile in the bright spot may differ from that of neighboring points. Such a variation could also account for the observed enhancement of $\mathrm{C}_{2} \mathrm{H}_{2}$ emission as discussed in the following section.

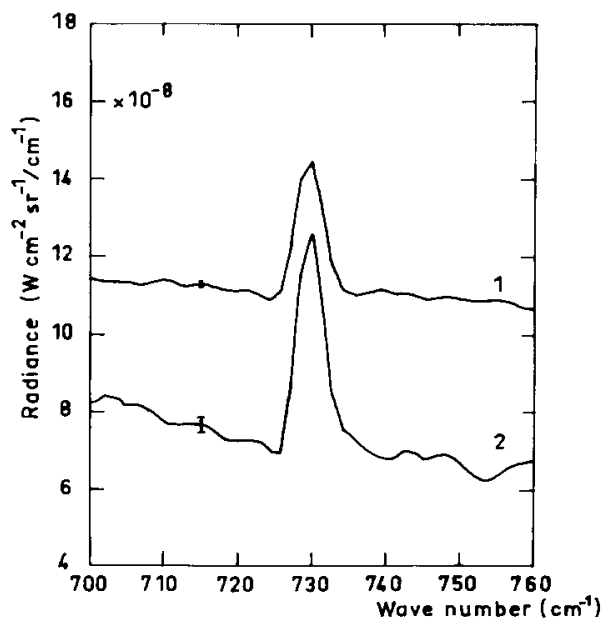

FiG. 5. Voyager spectra in the acetylene $\nu_{5}$ band, corresponding to selections 1 (equatorial) and $2\left(60^{\circ}\right.$ North) of Fig. 4. 


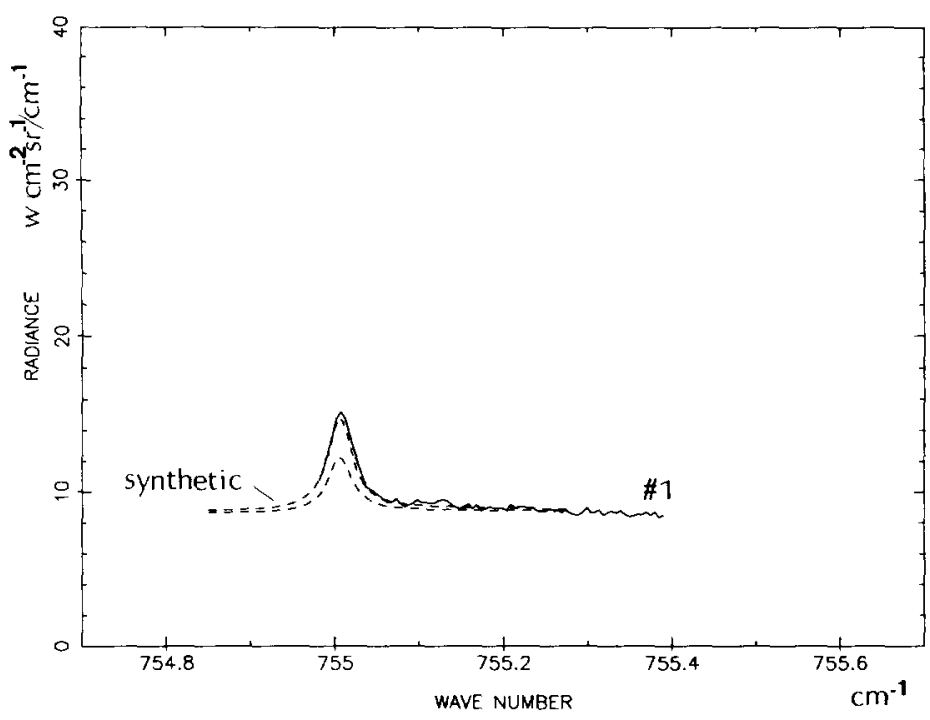

FIG. 6. Synthetic calculation (dashed line) for spectrum 1 (radiances are in $\mathrm{W} \mathrm{cm}^{-2} \mathrm{sr}^{-1} / \mathrm{cm}^{-1} \times 10^{8}$ ). Column abundances are (1) $8 \times 10^{-4} \mathrm{~cm} \cdot$ am (upper); (2) $4 \times 10^{-4} \mathrm{~cm} \cdot$ am (lower).

The value of the continuum within the bright spot is found to be about $10 \%$ lower than the same latitude outside the bright spot. This difference is significantly above the noise level. Since the bright spot is near the northern limb of Jupiter, it is possible that part of the beam was off the planet's disk, resulting in a lower continuum. However, this effect may be real, and can be interpreted in the synthetic spectrum by an absorption in a layer whose temperature is lower than the continuum temperature (about $123 \mathrm{~K}$ ). This layer could be an aerosol layer in the tropopause vicinity, giving a

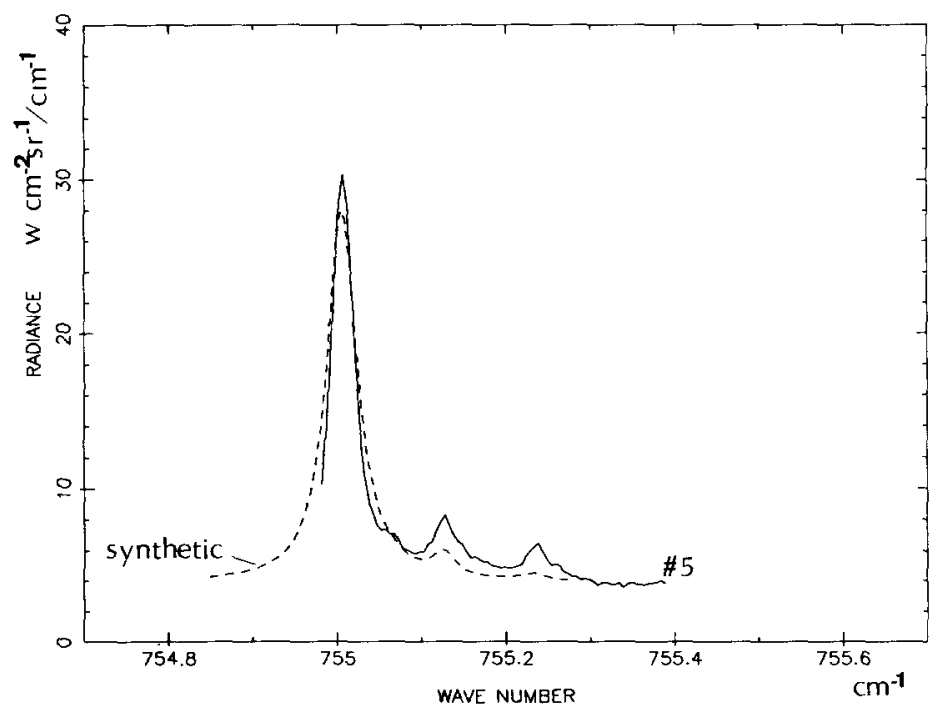

FIG. 7. Synthetic calculation (dashed line) for the bright spot (spectrum 5). The column abundance is $8 \times 10^{-3} \mathrm{~cm} \cdot$ am. (Radiances are in $\mathrm{W} \mathrm{cm}-2 \mathrm{sr}^{-1} / \mathrm{cm}^{-1} \times 10^{8}$.) 


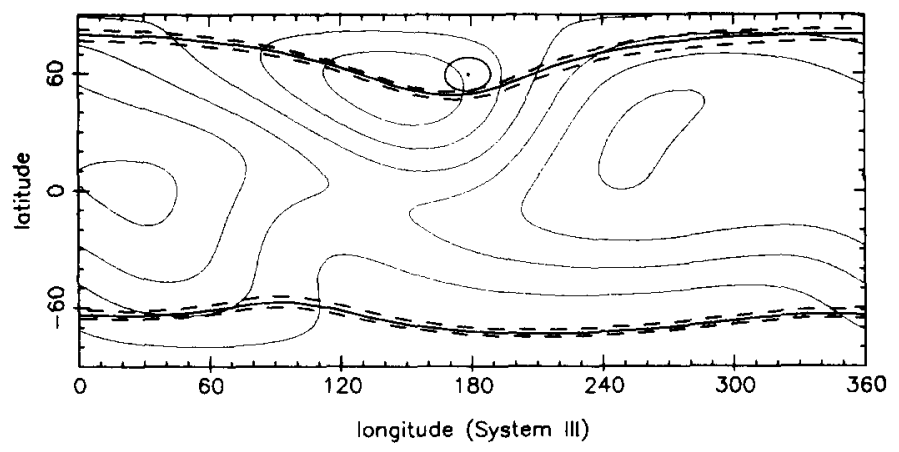

FIG. 8. Comparison of the positions of the bright spot with the magnetic anomaly (Dessler et al. 1979). The magnetic field representation is derived from the $\mathrm{O}_{4}$ model of Acuna and Ness (1976). The solid lines north and south are the foot of the magnetic field lines crossing Io; the dashed lines are the foot of the Io torus lines. The circular field of the view of the instrument is plotted at the position of the bright spot (latitude of $60^{\circ}$, longitude of $180^{\circ}$ ).

greater absorption coefficient in the bright spot than outside (attenuation factor $\tau=\mathbf{0 . 1}$ for an aerosol layer located at the tropopause).

\section{INTERPRETATION}

The observed location of the bright spot on Jupiter, latitude $59 \pm 10^{\circ}$ and longitude $178 \pm 10^{\circ}$, is very close to the region where intense auroral emissions of $\mathrm{H}_{2}$ Lyman and Werner bands and $\mathrm{H}$ Lyman- $\alpha$ were detected by the Voyager UV spectrometer (Broadfoot et al., 1979, 1981). In fact, at the longitude corresponding the bright spot, the UV data show auroral emissions at latitudes of $56+4 /-3$ degrees (see, e.g., Fig. 20 in Broadfoot et al. 1981). These latitudes lie in the same range as those of the bright spot reported here. It should be remarked that the exact location of the UV aurora seen by Voyager is uncertain by a few degrees due to the fact that the aurorae are near the polar limb and the UVS slit has a finite dimension of $0.1 \times 1^{\circ}$. For example, the equatorward boundary of the auroral zone corresponding to the longitude of the bright spot of this paper is found to be at 54 $\pm 1^{\circ}$ latitude, whereas the equatorward boundary of the magnetic mapping of the Io plasma torus on Jupiter lies between latitudes 50 and $53^{\circ}$ (slightly removed from the auroral boundary as expected). In any event, the possible latitude range for the bright spot is easily accommodated within the UV auroral zone even after accounting for all its positioning uncertainties.

The bright spot is also found at a location within the magnetic anomaly reported by Acuna and Ness (1976), as is shown in Fig. 8. This magnetic anomaly could be associated with enhanced particle precipitations.

The charged particle precipitation in the auroral zone can have major consequences of relevance to the present $\mathrm{C}_{2} \mathrm{H}_{2}$ observations. First, it could result in increased production of $\mathrm{C}_{2} \mathrm{H}_{2}$ due to particle dissociation of $\mathrm{CH}_{4}$, and, second, it could cause an increase in the stratospheric temperature. If the UV aurorae are caused by precipitation of energetic electrons, their penetration can be to greater depths in the Jovian atmosphere than in the case where energetic ions might be responsible for the auroras. The former are expected to result in enhanced abundances of the hydrocarbon byproducts (e.g., $\mathrm{C}_{2} \mathrm{H}_{2}$ ) following particle dissociation of $\mathrm{CH}_{4}$. The observation of a bright spot reported here could very well be the consequence of such enhanced $\mathrm{C}_{2} \mathrm{H}_{2}$ abundance. Another consequence of increased $\mathrm{C}_{2} \mathrm{H}_{2}$ abundance is the possibility of greater concentration of polymers which are expected to form subsequent to the photolysis of $\mathrm{C}_{2} \mathrm{H}_{2}$. The polymers would ul- 
timately condense and fall out of the stratosphere. They would re-evaporate once below the tropopause due to the warmer temperatures prevalent there. This polymer layer could constitute the aerosol layer which is suggested to explain the continuum level of the bright spot (see Section III).

Precipitation of heavy ions, such as those of sulfur and oxygen, has been proposed by Gehrels and Stone (1983) on the basis of Voyager cosmic-ray instrument data. The heavy ions presumably have their origin in the Io plasma torus, and ultimately in the volcanic outgassing from Io. Because of their shorter range, these ions are expected to deposit their energy near or above the microbar level on Jupiter. The estimated power input due to energetic ions at Jupiter is $10^{12}$ to $10^{13}$ (Gehrels and Stone, 1983), or approximately 1 to $10 \mathrm{erg} \mathrm{cm}^{-2} \mathrm{sec}^{-1}$ in the auroral zone. Such a large energy flux would have important consequences for the Jovian thermal structure. Although the effect on the stratospheric temperature cannot be calculated exactly since the "normal" thermal structure and the heat loss mechanisms in the middle atmosphere are not known precisely, it is reasonable to expect some rise in the stratospheric temperature-purely on the basis of heat conduction from above. In fact, only a $10 \mathrm{~K}$ increase in temperature at the $1-\mathrm{mb}$ level is needed to explain the observed bright spot. Such a rise in the temperature would require less than $0.03 \mathrm{erg} \mathrm{cm}^{-2} \mathrm{sec}^{-1}$ additional heat input as the $1-\mathrm{mb}$ level, which is well within the realm of possibility of conduction of auroral energy from above.

Our conclusions are in agreement with the results found by Kim et al. (1985), from the analysis of IRIS spectra.

\section{CONCLUSIONS}

The observation of a polar bright spot in the atmosphere of Jupiter is characterized by an enhancement in the individual lines of $\mathrm{C}_{2} \mathrm{H}_{2}$ which can be interpreted as an enhancement in the acetylene abundance, al- though a purely thermal effect, or non-LTE phenomena cannot be excluded. The intensity of the observed hot band lines are also consistent with either hypothesis. The source of the anomaly could be due to particle precipitation, as in the UV aurorae. The penetration of the particles (most likely secondary electrons), or the heat deposition has to be as deep as the lower stratosphere where the acetylene lines are formed.

An unambiguous interpretation would require observing other molecules (such as $\mathrm{C}_{2} \mathrm{H}_{6}$, for example) to discriminate between photochemical and thermal effects. The chemistry of ethane in the stratosphere is different from the chemistry of acetylene, and the modifications to both cannot be the same if the effect is only in a modification of the abundances.

Finally, a measurement of the rotational temperature of $\mathrm{C}_{2} \mathrm{H}_{2}$, obtained by recording various rotational levels of different $J$, or by recording fully resolved line profiles, is required to determine the importance of nonequilibrium phenomena on the emission lines of $\mathrm{C}_{2} \mathrm{H}_{2}$.

\section{ACKNOWLEDGMENTS}

S. Atreya's contribution was sponsored by a grant from NASA Planetary Atmospheres Program (work done while on sabbatical at Université de Paris). P. Drossart thanks F. Genova (Paris-Meudon Observatory) for providing information on the Jovian magnetic field (Fig. 8). J. Lacy and E. Serabyn were supported by NASA grant NGL 05-003-272.

\section{REFERENCES}

ACUNA AND Ness (1976). The main magnetic field of Jupiter. J. Geophys. Res. 81, 2917-2922.

AtreyA, S. K. (1984). Aeronomy. In Uranus and Neptune (J. T. Bergstrahl, Ed.), NASA.CP 2330.

Atreya, S. K., AND P. N. Romani (1985). Photochemistry and clouds of Jupiter, Saturn and Uranus. In Planetary Meteorology (G. E. Hunt, Ed.), Cambridge Univ. Press, London/New York.

Beck, S. C., E. E. Bloemhof, E. Serabyn, C. H. Townes, A. T. Tokunaga, J. H. LaCy, and H. A. SMIтH (1982). High spectral and spatial resolution observations of the 12.28 micron emission from $\mathrm{H}_{2}$ in the Orion molecular cloud. Astrophys. J. 253, L83-L87.

Broadfoot, A. L., M. J. S. Belton, P. Z. Takacs, B. R. Sandel, D. E. Shemansky, J. B. Holberg, 
J. M. Ajello, S. K. Atreya, T. H. Donahue, H. W. Moos, J. L. Bertaux, J. E. Blamont, D. F. Strobel, J. C. McConnell, A. Dalgarno, R. GoOdY, AND M. B. MCELROY (1979). Extreme ultraviolet observations from Voyager 1 encounter with Jupiter. Science 204, 979-982.

Broadfoot, A. L., B. R. Sandel, D. E. Shemansky, J. C. McConnell, G. R. Smith, J. B. Holberg, S. K. Atreya, T. H. Donahue, D. F. Strobel, and J. L. Bertaux (1981). J. Geophys. Res. 86, 8259-8284.

Caldwell, J., A. T. Tokunaga, and F. C. Gillett (1980). Possible infrared aurorae on Jupiter. Icarus 44, 667-675.

Caldwell, J., A. T. Tokunaga, and G. S. Orton (1983). Further observations of $8 \mu \mathrm{m}$ Polar brightenings of Jupiter. Icarus 53, 133-140.

Combes, M., T. Encrenaz, L. Vapillon, Y. Zeau, AND C. Lesqueren (1974). Confirmation of the identification of $\mathrm{C}_{2} \mathrm{H}_{2}$ and $\mathrm{C}_{2} \mathrm{H}_{6}$ in the Jovian atmosphere. Astron. Astrophys. 34, 33.

Dessler, A. J., and J. W. Chamberlain (1979). Jovian longitudinal asymmetry in Io related and Europa related auroral hot spots. Astrophys. J. 230, 974-981.

Dessler, A. J. (1983). Physics of the Jovian Magnetosphere. Cambridge Univ. Press, New York.

Drossart, P., T. Encrenaz and M. Combes (1985a). The ${ }^{14} \mathrm{~N} /{ }^{15} \mathrm{~N}$ ratio in the Jovian atmosphere. Astron. Astrophys. 146, 181-184.

Drossart, P., J. Lacy, E. Serabyn, A. T. Tokunaga, B. BEzard, AND T. EnCREnaz (1985b). Detection of ${ }^{13} \mathrm{C}^{12} \mathrm{CH}_{2}$ on Jupiter at $13 \mu \mathrm{m}$. Astron. Astrophys. 149, L10-L12.

GAUTIER et al. (1977). The thermal structure of Jupiter from infrared spectral measurements by means of a filtered iterative inversion method. J. Atmos. Sci. 34, 1130-1137.

Gautier, D., B. Bezard. A. Marten, J. P. Baluteau, N. Scott, A. Chédin, V. Kunde, and R. HANEL (1982). The C/H ratio in Jupiter from the Voyager infrared investigation. Astrophys. J. 257, 901-912.

GAUTIER AND OWEN (1983). Nature 304, 691-694.

Gladstone, G. R. (1982). Thesis, California Institute of Technology.

Hanel, R., B. Conrath, M. Flasar, V. Kunde, P. Lowman, W. Maguire, J. Pearl, J. Pirraglia, R. Samuelson, D. Gautier, P. Gierasch, S. KuMAR, AND C. PONNAMPERUMa (1979). Infrared ob- servations of the Jovian system from Voyager 1. Science 204, 972.

Hietanen. J., and J. Kaupinnen (1981). High resolution infrared spectrum of acetylene in the region of the bending fundamental $\nu_{5}$. Mol. Phys. 42, 411423.

Kim, S. J., J. Caldwell, A. R. Rivolo, R. WAGENER, AND G. S. ORTON (1985). Infrared polar brightening on Jupiter III. Spectrometry from the Voyager I IRIS experiment. Icarus, to be published.

Kostiuk, T., M. J. Mumma, J. J. Hillman, D. BuHL, L. W. Brown, AND J. L. FarIs (1977). $\mathrm{NH}_{3}$ spectral line measurements on earth and Jupiter using a $10 \mu \mathrm{m}$ superheterodyne receiver. Infrared Phys. 17, 431-439.

Kostiuk, T., M. J. Mumma, F. Espenak, D. Deming, D. E. Jennings, ANd W. Maguire (1983). Measurements of the stratospheric ethane in the Jovian south polar region from infrared heterodyne spectroscopy of the $\nu_{9}$ band near $12 \mu \mathrm{m}$. Astrophys. J. 265, 564-569.

Maguire, W. C., R. E. Samuelson, R. A. Hanel, AND V. G. KUNDE (1984). Latitudinal variation of the acetylene and ethane in the Jovian atmosphere from Voyager IRIS observation. Bull. Amer. Astron. Soc. 16, 647.

Orton, G. S., et al. (1984). Relative temperature fields in the Jovian atmosphere in 1984. Bull. Amer. Astron. Soc. 16, 641.

Ridgway, S. T. (1974). Jupiter: Identification of ethane and acetylene. Astrophys. J. 187, L41; erratum 192, L51.

Serabyn, E. (1984). Ph.D. Thesis, University of California, Berkeley.

Strobel, D. F. (1974). Hydrocarbon abundances in the Jovian atmosphere. Astrophys. J. 192, L47.

Tokunaga, A., R. F. KNacks, S. T. RidgWay, AND L. WALLACE (1979). High resolution spectra of Jupiter in the 744-980 inverse centimeter spectral range. Astrophys. J. 232, 603-615.

Tokunaga, A., R. F. Knacke, and T. Owen (1976). Ethane and acetylene abundances in the Jovian at mosphere. Astrophys. J. 209, 294-301.

Tokunaga, A. T., S. C. Deck, T. R. Geballe. J. H. LACY, AND E. SERABYN, (1981). The detection of HCN on Jupiter. Icarus 48, 283-289.

Varanasi, P., P. Giver, and F. P. J. Valero (1983). Infrared absorption by acetylene in the $12-14 \mathrm{mi}$ cron region at low temperatures. J. Quant. Spectrosc. Radiat. Transfer 30, 497-504. 